\title{
A Case of Actinomycosis Causing Unilateral Tonsiller Hypertrophy
}

Kenji Takasaki, $\mathrm{MD}^{1)}$, Kyoko Kitaoka, $\mathrm{MD}^{1)}$,

Satoru Kaieda, $\mathrm{MD}^{1)}$, Tomayoshi Hayashi, $\mathrm{MD}^{2)}$,

Kuniko Abe, $\mathrm{MD}^{2)}$, Haruo Takahashi, $\mathrm{MD}^{1)}$

1) Division of Otorhinolaryngology,

Department of Translational Medical Sciences,

Nagasaki University Graduate School of Biomedical Sciences

2) Department of Pathology,

Nagasaki University Hospital

Address:

Division of Otorhinolaryngology,

Department of Translational Medical Science,

Nagasaki University Graduate School of Biomedical Sciences

1-7-1, Sakamoto, Nagasaki 852-8501, Japan

Correspondence / Reprint requests:

Kenji Takasaki, MD

Division of Otorhinolaryngology,

Department of Translational Medical Science,

Nagasaki University Graduate School of Biomedical Sciences

1-7-1, Sakamoto, Nagasaki 852-8501, Japan

Phone: +81-95-849-7349, Fax: +81-95-849-7352

E-mail: ktakasa@net.nagasaki-u.ac.jp 


\begin{abstract}
We report a case of unusual presentation of actinomycosis in the tonsil causing massive unilateral enlargement in a 78-year-old female. To our knowledge, only three cases of actinomycosis causing unilateral tonsillar enlargement have previously been published. Since this anaerobic organism is difficult to culture, the diagnosis is made by observing its associated sulfur granules in the biopsy specimen. In the present case, treatment consisting of tonsillectomy and antibiotic therapy (penicillin) for several days revealed a good prognosis.
\end{abstract}

Key Words: unilateral tonsillar enlargement, actinomycosis 


\section{Introduction}

Actinomycosis is a disease, which is not frequently encountered in these days because of widespread use of antibiotics. To our knowledge, there were only three cases of the unilateral tonsillar hypertrophy caused by actinomycosis reported. Two cases of them demonstrated masquerading as a tumor $(1,2)$ and the remainder revealed a pendulous tonsil (3). The incidence of the presence of actinomycosis in tonsils has recently reported to be was $18.3 \%$ (4) and 28.5\% (5). However these reports did not state whether unilateral tonsillar hypertrophy could be caused by existence on actinomycosis or not. We recently experienced and herewith report a rare case with actinomycosis causing unilateral massive hypertrophy of the tonsil.

\section{Case report}

A 75-year-old woman was referred to our outpatient clinic of Nagasaki University Hospital, complaining of pharyngeal discomfort for one month. She had no episodes of fever or sore throat. Oral and pharyngeal examination showed enlargement of the left-side tonsil with normal mucosa and atrophy of the right-side tonsil (Figure 1). Left-side tonsil was soft on palpation. There was no other abnormal finding in her head and neck on the physical examination. Her blood examination revealed no abnormality either. Computed tomography (CT ) revealed a soft-tissue-density mass with enhancement arising from the left-side tonsil (Figure 2). 
Despite administration of one-week oral antibiotics (Levofloxacin), the size of her left-side tonsil has even increased. Suspecting malignant lymphoma, biopsy of the left-side tonsil was performed, showing nonspecific inflammation.

We underwent bilateral tonsillectomy under general anesthesia (Figure 3). Histological examination of her left-side tonsil demonstrated reactive hyperplasia with Actinomyces in dilated crypts (Figure 4, 5). Many lymphocytes demonstrated immunoreactivity for Ki-67 (DakoCytomation Corp.), indicating proliferating cells, and lymphocytes mainly located in the lymphoid follicle showed immunoreactivity for L-26 (NICHIREI Corp.), detecting B-lymphocytes (Figure 6, 7). On the other hand her right-side tonsil demonstrated fatty degeneration and fibrosis without findings of Actinomyces histopathologically (Figure 8). A diagnosis of tonsiller actinomycosis was made, although bacteriological examination of the left-side tonsil during surgery showed no growth of any microorganism. She was treated with penicillin for 3 days intravenously and subsequently for 4 days orally. Seven months after treatment now, she is well without signs of recurrence.

\section{Discussion}

Morikawa et al. (1) stated histopathological features of tonsiller actinomycosis as follow; 1) invasion of the germ lump to inside the lymphoid follicle, 2) infiltration of inflammatory cells around the germ lump, 3) a radical spokelike fashion on the outside layer of germ lump (to defense tact of the germ for the landlord 
immune reaction). In the present case, although her right-side tonsil showed atrophic change (fatty degeneration and fibrosis) due to aging (6), her left-side tonsil demonstrated reactive lymphoid hyperplasia with typical histopathologial features of tonsillar actinomycosis mentioned above. Furthermore, since Ki-67 positive cells and L-26 positive cells were observed in her left-side tonsil, we speculate that B-lymphocytes proliferate to produce the antibodies against actinomycosis. However we did not know why actinomycosis caused tonsillar hypertrophy only on the left side. Actinomyces is now known to be a gram-positive, anaerobic or microaerophilic bacterium that grows as a hyphae or filamentous organism, although it had previously been referred to as a fungus. It is distinguished from Nocardia in that it is non-acid-fast. They are anaerobic or microaerophilic and difficult to culture. Recovery rates from culture are less than $30 \%$, due to a low index of suspicion, lack of proper culture conditions, and the fastidious nature of the organism (7).

Actinomycosis has been identified in numerous locations in the body, including the oral cavity, pharynx, dental plaques, tonsillar crypts, carious dentin, gingiva1 surface, and periodontal pockets (8). Actinomycosis acts as a commensal organism and is unable to penetrate healthy tissues to cause infection, indicating that there should be a portal of entry into unhealthy or disturbed tissue for infection to develop. However, we should keep in mind that actinomycosis acts as pathogenetic agent of tonsillar hypertrophy even in healthy patients as the present case.

CT findings in actinomycocis of the head and neck are nonspecific. CT demonstrates a soft tissue mass with the intensity similar to or slightly lower than 
adjacent muscles. The mass may have a well-defined rim or capsule with low attenuation in the central area (9). Although not highly diagnostic, thus CT can be of help in distinguishing the lesion from the malignancy, and in delineating the extent of the lesion. This may also be of great help in surgery.

An empirical trial of high-dose penicillin for 1 to 12 months as a medical management of actinomycosis has been suggested. Reports have also documented success with clindamycin, tetracychne, and sulfonamides (10). Hyperbaric oxygen has also been used in one report as adjuvant treatment because of its direct bactericidal effect (8). Classical surgical excision is thought as a definitive treatment for head and neck actinomycosis, and tonsillectomy is usually curative for tonsillar actinomycosis (5). Therefore, we did not add any treatment to tonsillectomy aexpect penicillin only in one week.

Although actinomycosis can be detected in the tonsil comparatively frequently as mentioned above, there is few report that actinomycosis is relevant to unilateral tonsillar hypertrophy. Insufficient recognition of the unilateral tonsillar hypertrophy caused by actinomycosis among otolaryngologists may be one of the possible reasons.

\section{References}

1. Morikawa I, Shin T, Watanabe H, Matsuo K, Yukizane K. Actinomycosis originating from palatine tonsil and its diagnosis. (in Japanese) Otologia Fukuoka 1985;31:382-386. 
2. Yadav SPS, Chanda R, Gathwala G, Yadav RK. Actinomycosis of tonsil masquerading as tumour in a 12-year old child. Int J Pediatr Otorhinolaryngol 2002;63:73-75.

3. Hirai S, Akisada T, Yoshihiro T, Take T, Orita Y. A case report of a pendulous tonsil induced by Actinomyces infection. (in Japanese) Pract Otol (Kyoto) 1998;suppl 96:146-148.

4. Pransky SM, Feldman JI, Kearns DB, Seid AB, Billman GF. Actinomycosis in obstructive tonsillar hypertrophy and recurrent tonsillitis. Arch Otolaryngol Head Neck Surg 1991;117:883-885.

5. Bhargava D, Bhusnurmath B, Sundaram KR, Raman R, Okbi HMA Abri RA, Date A. Tonsillar actinomycosis: a clinicopathological study Acta Trop. 2001;80:163-168.

6. Ikeda M. Histopathological study on the chronological change of human palatine tonsils. (in Japanese) Nippon Jibiinkoka Gakkai Kaiho. 1975;78:623-637.

7. Brown JR. Human actinomycosis: a study of 181 subjects. Human Pathol. 1973;4:319-330.

8. Nagler R Peled M, Laufer D. Cervicofacial actinomycosis: a diagnostic challenge. Oral Surg Oral Med Oral Pathol 1997;83:652-656.

9. Allen H, Scatarige JC, Kim MH. Actinomycosis: CT finding in six patients . AJR 1987;149:1255-1258.

10. Bennhoff DF. Actinomycosis: diagnostic and therapeutic considerations and a review of 32 cases. Laryngoscope 1984;94:1198-1217. 


\section{Legend}

Figure 1

Oral examination showed hypertrophy of the left-side tonsil $\left(^{*}\right)$ beyond the medial line. Arrows show palatoglossal arch.

Figure 2

Computed tomography scan of oropharynx demonstrates a soft tissue mass with enhancement arising from left-side tonsil $(*)$.

Figure 3

Gross appearances of resected tonsils demonstrating the left-side tonsil covered with normal mucosa with $35 \times 30 \times 20 \mathrm{~mm}$ in size.

Figure 4

Histopathological picture of resected left-side tonsil (H \& E, x 6.6). Sulfur granule of Actinomyces (*) exists within tonsillar crypts among hyperplastic lymphoid follicle ( $\mathcal{\sim})$

Figure 5

Histopathological picture of resected left-side tonsil (H \& E, x 132).

Aggregates of basophilic, filamentous Actinomyces locates within tonsillar crypts and neutrophils envelopes.

Figure 6

Immunohistochemical picture of resected left-side tonsil (x 66).

Ki-67 (DakoCytomation Corp.) positive cells were stained dark on lymphocytes

(arrows). *: Sulfur granule of Actinomyces

Figure 7 
Immunohistochemical picture of resected left-side tonsil (x 33).

L-26 (NICHIREI Corp.) positive cells were stained dark and mainly located in lymphoid folliles (arrows). *: Sulfur granule of actinomycetes

Figure 8

Histopathological picture of resected right-side tonsil (H \& E, x 6.6).

Right-side tonsil demonstrated fatty degeneration (arrows) and fibrosis (F). 


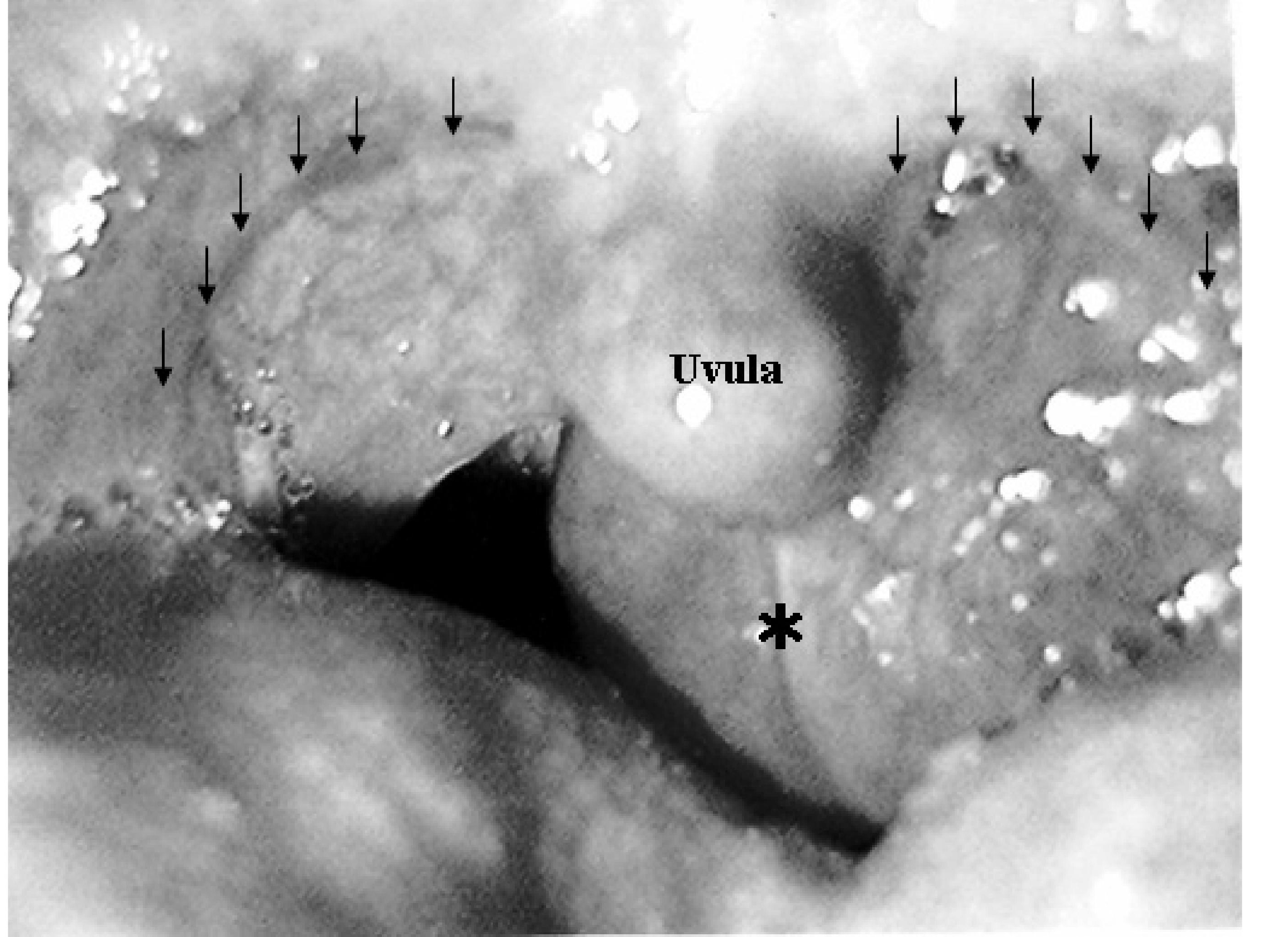




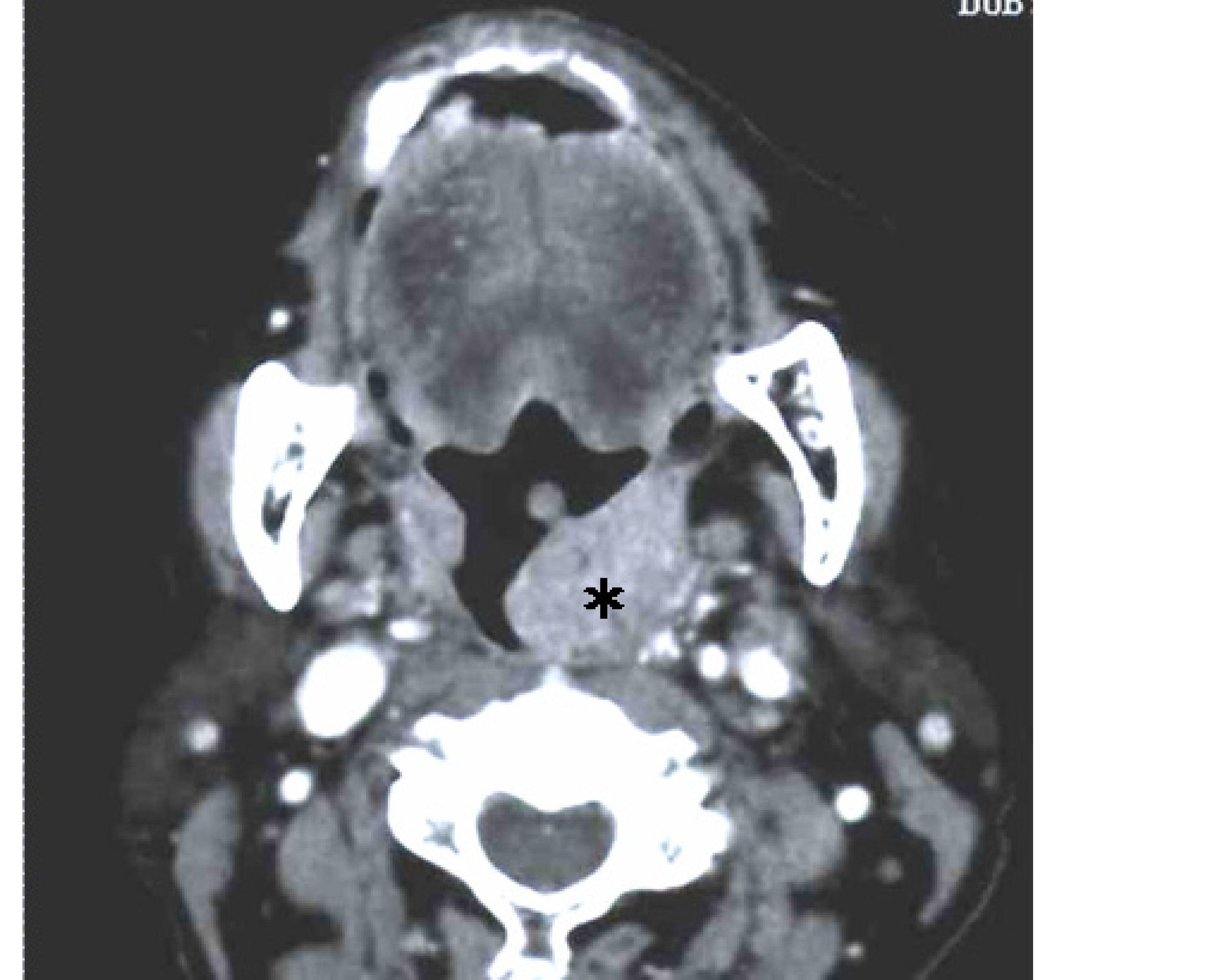




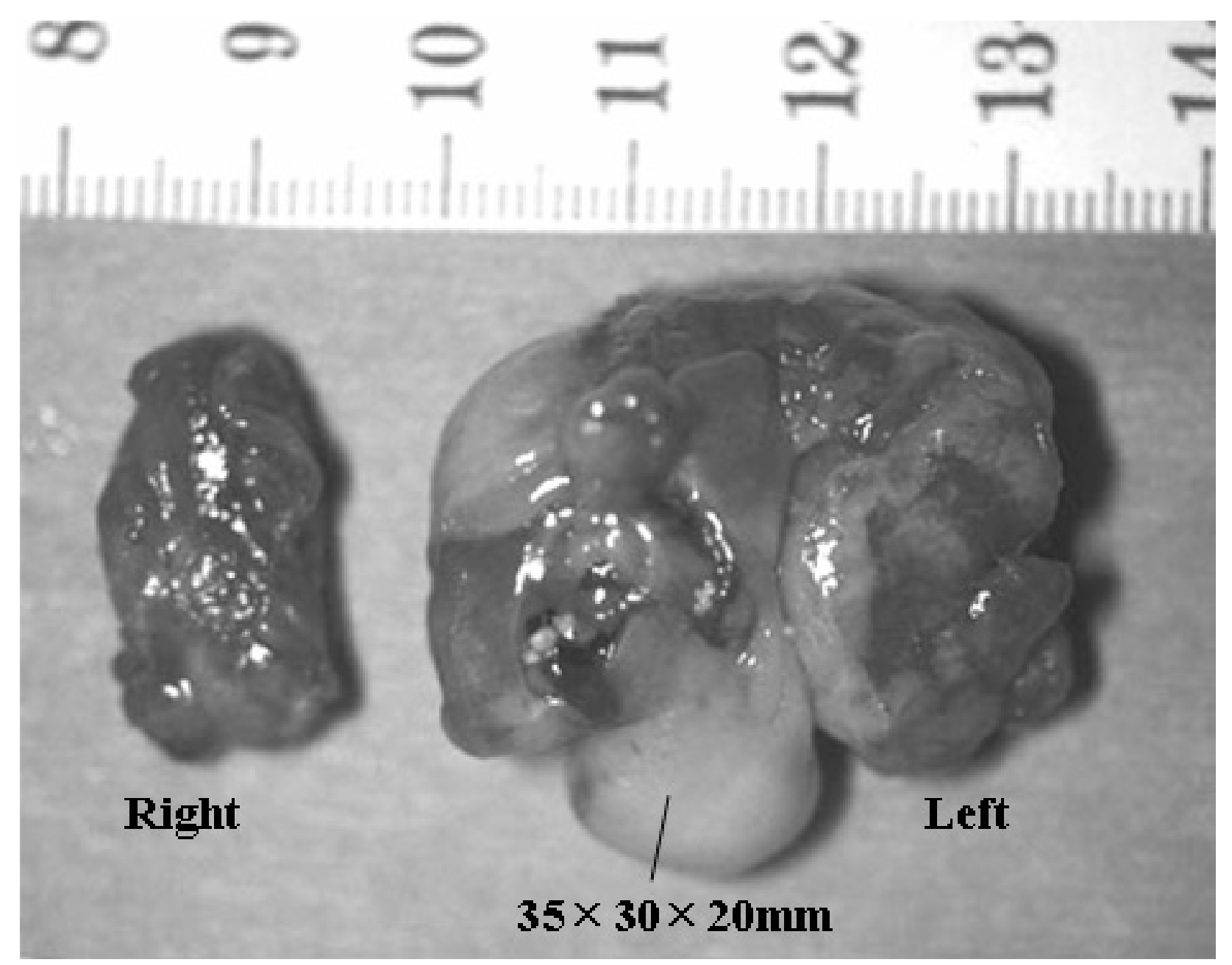




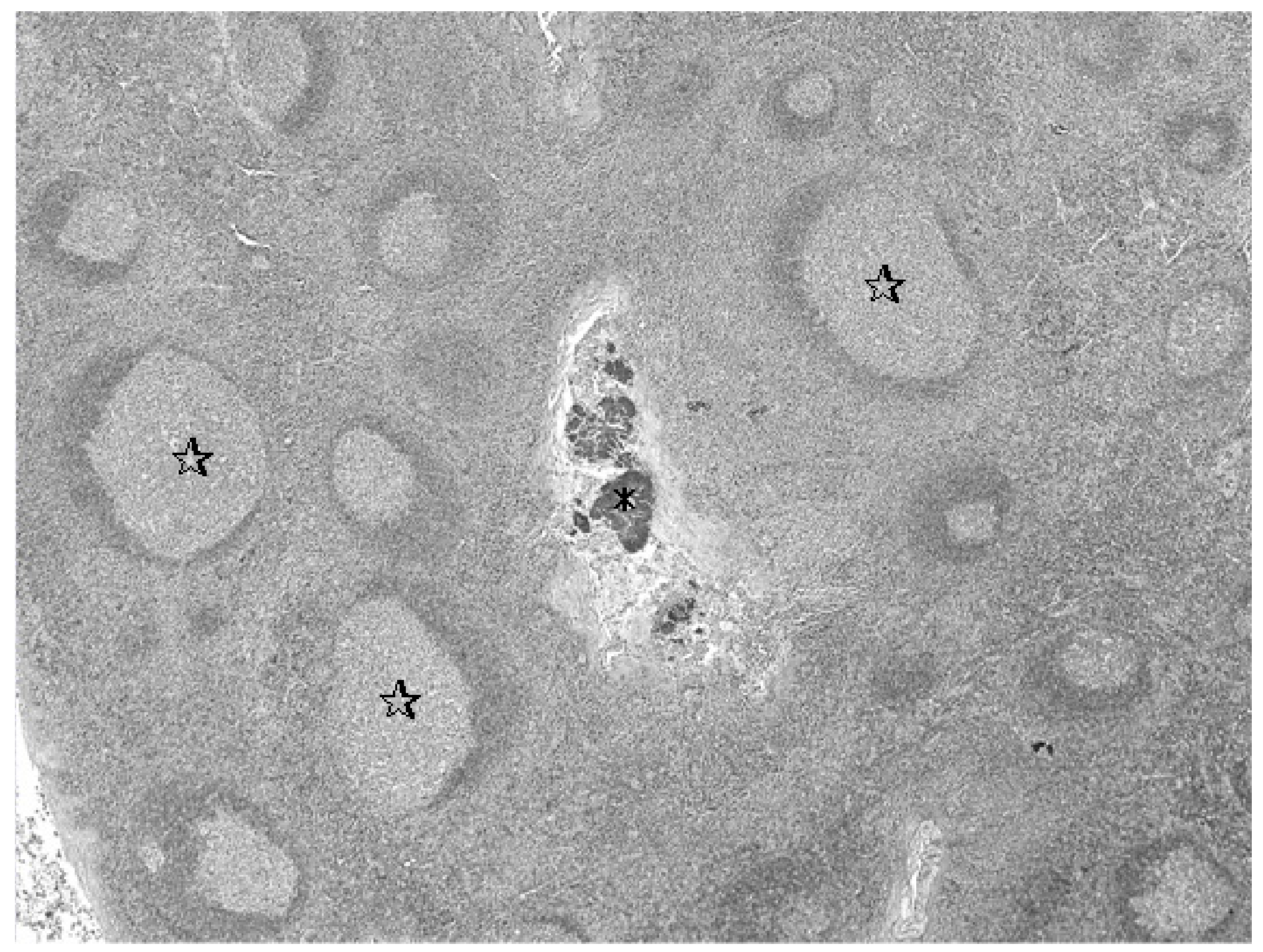




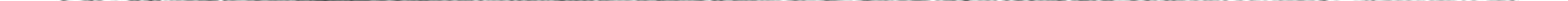




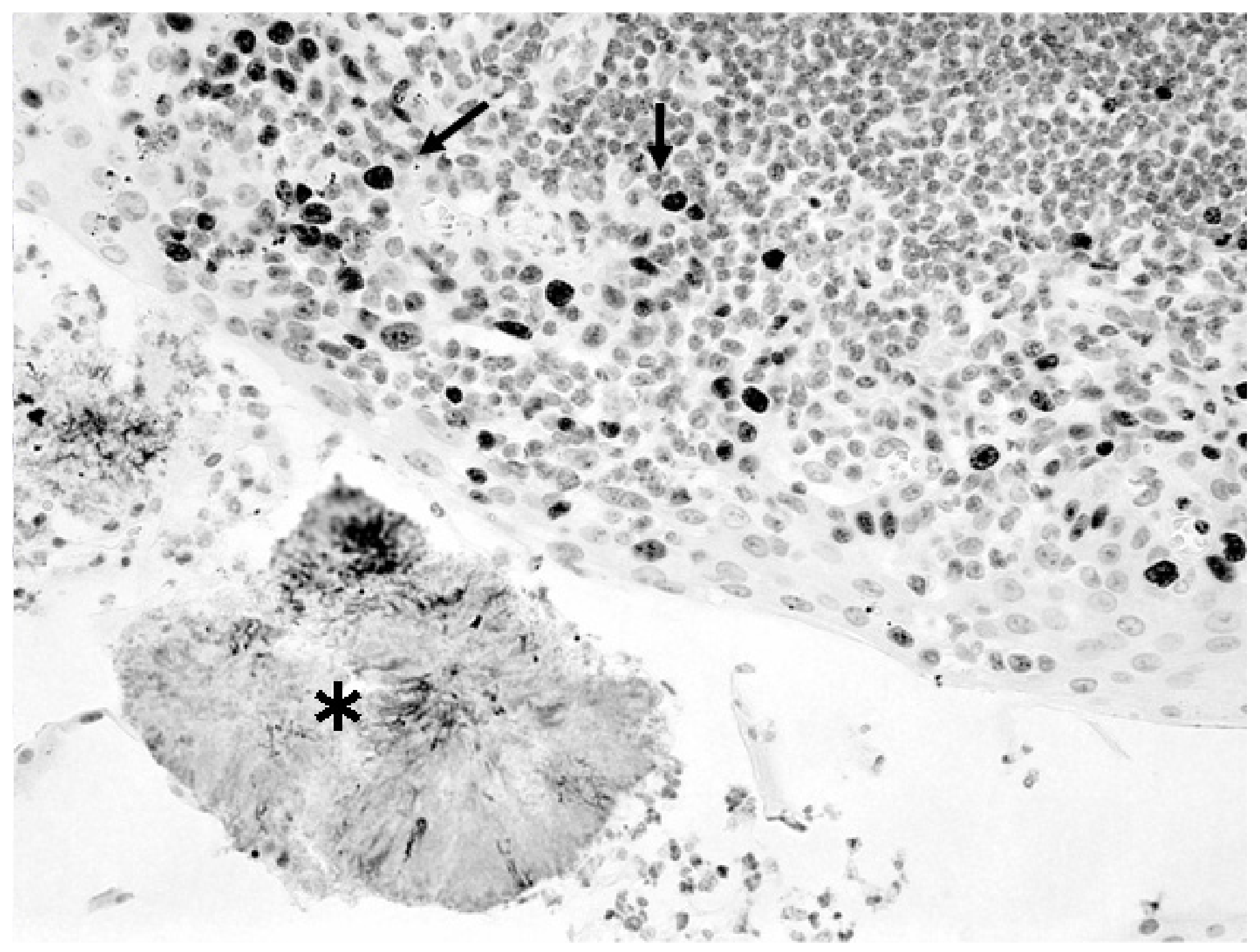




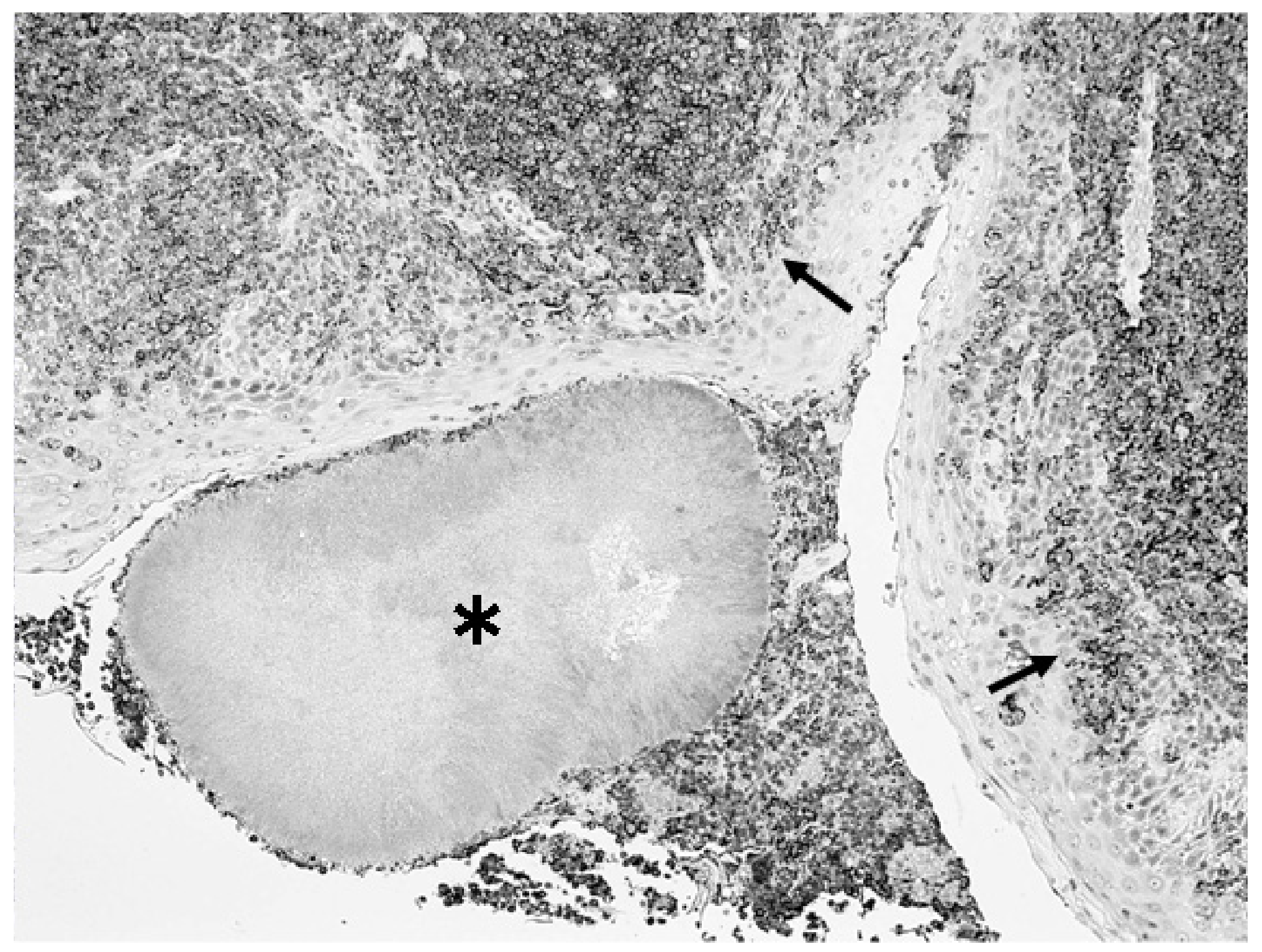




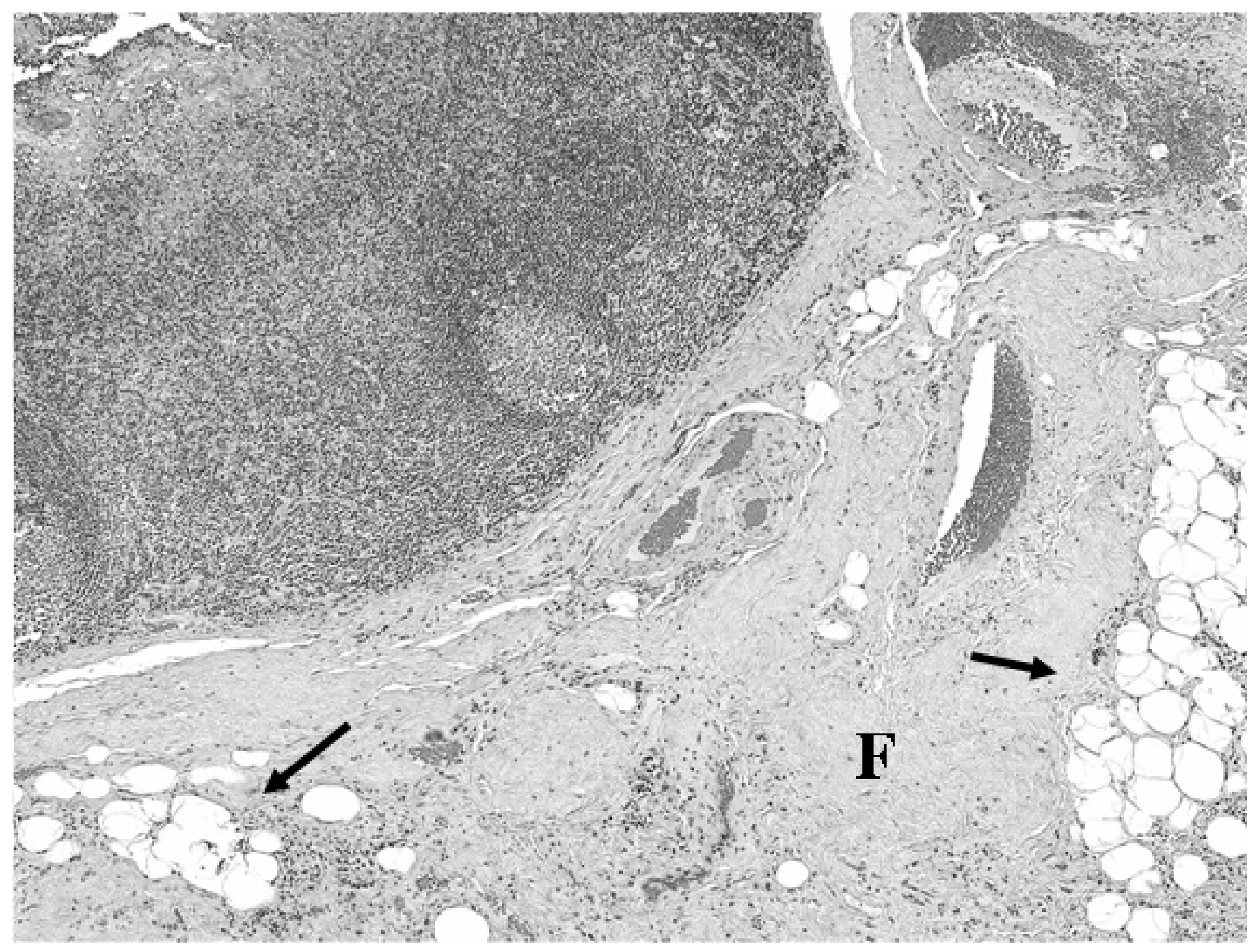

